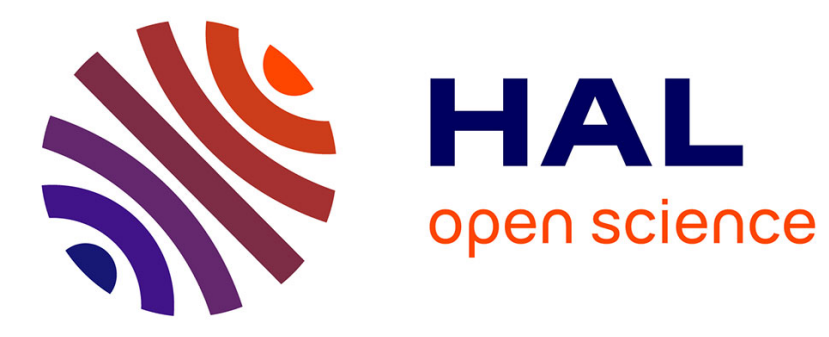

\title{
Static Analysis of Endian Portability by Abstract Interpretation
}

\author{
David Delmas, Abdelraouf Ouadjaout, Antoine Miné
}

\section{To cite this version:}

David Delmas, Abdelraouf Ouadjaout, Antoine Miné. Static Analysis of Endian Portability by Abstract Interpretation. 28th Static Analysis Symposium (SAS 2021), Oct 2021, Chicago, Illinois, United States. pp.102-123, 10.1007/978-3-030-88806-0_5 . hal-03450165

\section{HAL Id: hal-03450165 \\ https://hal.sorbonne-universite.fr/hal-03450165}

Submitted on 25 Nov 2021

HAL is a multi-disciplinary open access archive for the deposit and dissemination of scientific research documents, whether they are published or not. The documents may come from teaching and research institutions in France or abroad, or from public or private research centers.
L'archive ouverte pluridisciplinaire HAL, est destinée au dépôt et à la diffusion de documents scientifiques de niveau recherche, publiés ou non, émanant des établissements d'enseignement et de recherche français ou étrangers, des laboratoires publics ou privés. 


\title{
Static Analysis of Endian Portability by Abstract Interpretation ${ }^{\star}$
}

\author{
David Delmas $^{1,2} \odot$, Abdelraouf Ouadjaout ${ }^{2} \odot$, and Antoine Miné ${ }^{2,3} \odot$ \\ 1 Airbus Operations S.A.S., 316 route de Bayonne, 31060 Toulouse Cedex 9, France \\ 2 Sorbonne Université, CNRS, LIP6, 75005 Paris, France \\ 3 Institut universitaire de France, 1 rue Descartes, 75231 Paris Cedex 5, France \\ david.delmas@airbus.com, antoine.mine@lip6.fr, \\ abdelraouf .ouadjaout@lip6.fr
}

\begin{abstract}
We present a static analysis of endian portability for C programs. Our analysis can infer that a given program, or two syntactically close versions thereof, compute the same outputs when run with the same inputs on platforms with different byte-orders, a.k.a. endiannesses. We target low-level $\mathrm{C}$ programs that abuse $\mathrm{C}$ pointers and unions, hence rely on implementation-specific behaviors undefined in the $\mathrm{C}$ standard.

Our method is based on abstract interpretation, and parametric in the choice of a numerical abstract domain. We first present a novel concrete collecting semantics, relating the behaviors of two versions of a program, running on platforms with different endiannesses. We propose a joint memory abstraction, able to infer equivalence relations between littleand big-endian memories. We introduce a novel symbolic predicate domain to infer relations between individual bytes of the variables in the two programs, which has near-linear cost, and the right amount of relationality to express (bitwise) arithmetic properties relevant to endian portability. We implemented a prototype static analyzer, able to scale to large real-world industrial software, with zero false alarms.
\end{abstract}

Keywords: Formal Methods · Abstract Interpretation · Abstract Domains · Static Analysis · C Programming Language · Portability · Endianness · Industrial Application
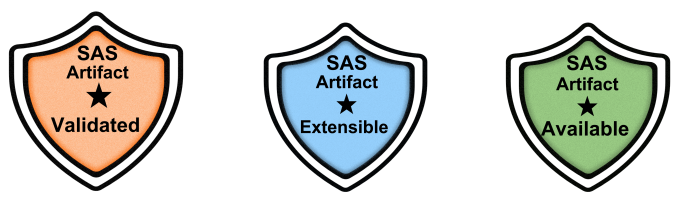

\footnotetext{
* This work is performed as part of a collaborative partnership between Sorbonnne Université / CNRS (LIP6) and Airbus. This work is partially supported by the European Research Council under the Consolidator Grant Agreement 681393 - MOPSA.
} 


\section{Introduction}

There is no consensus on the representation of a multi-byte scalar value in computer memory [10]. Some systems store the least-significant byte at the lowest address, while others do the opposite. The former are called little-endian, the latter big-endian. Such systems include processor architectures, network protocols and data storage formats. For instance, Intel processors are little-endian, while internet protocols and some legacy processors, such as SPARC, are big-endian. As a consequence, programs relying on assumptions on the encoding of scalar types may exhibit different behaviors when run on platforms with different byteorders, a.k.a. endiannesses. The case occurs typically with low-level C software, such as device drivers or embedded software. Indeed, the C standard [20] leaves the encoding of scalar types partly unspecified. The precise representation of types is standardized in implementation-specific Application Binary Interfaces (ABI), such as [3], to ensure the interoperability of compiled programs, libraries, and operating systems. Although it is possible to write fully portable, ABIneutral $\mathrm{C}$ code, the vast majority of $\mathrm{C}$ programs rely on assumptions on the ABI of the platform, such as endianness. Therefore, the typical approach used, when porting a low-level $\mathrm{C}$ program to a new platform with opposite endianness, is to eliminate most of the byte-order-dependent code, and to wrap the remainder, if any, in conditional inclusion directives, which results in two syntactically close endian-specific variants of the same program. A desirable property, which we call endian portability, is that a program computes the same outputs when run with the same inputs on the little- and big-endian platforms. By extension, we also say that a program is endian portable if two endian-specific variants thereof compute the same outputs when run with the same inputs on their respective platforms. In this paper, we describe a static analysis which aims at inferring the endian portability of large real-world low-level $\mathrm{C}$ programs.

Motivating example. For instance, Example 1 features a snippet of code for reading network input. The sequence of bytes read from the network is first stored into integer variable $\mathrm{x}$. Assume variable $\mathrm{y}$ has the same type. $\mathrm{x}$ is then either copied, or byte-swapped into y, depending on the endianness of the platform. Our analysis is able to infer that Example 1 is endian portable, i.e. both endianspecific variants compute the same value for $y$, whatever the values of the bytes read from the network. This property is expressed by the assertion at line 8 .

Example 1. Reading input in network byte-order.

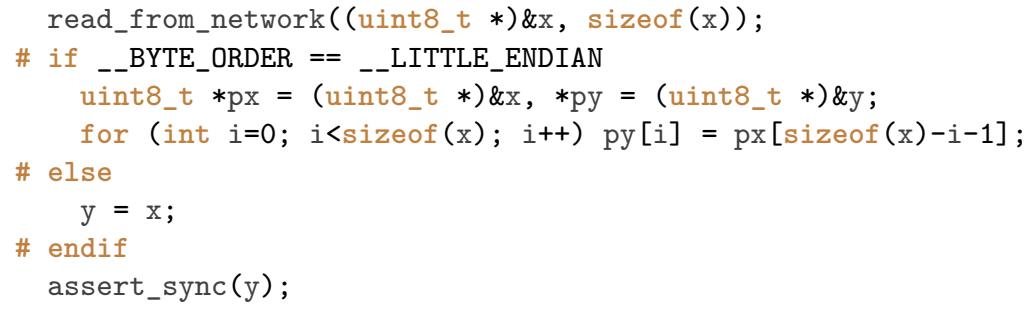


Example 1 abuses pointers to bypass the $\mathrm{C}$ type system, a common practice in low-level programming known as type punning. Alternatively, some implementations rely on bitwise arithmetics. E.g., if $\mathrm{x}$ and $\mathrm{y}$ have type uint32_t, the little-endian case may be rewritten as $((\mathrm{x} \& 0 \mathrm{xff000000})>>24) \mid((\mathrm{x}$ $\& 0 x f f 0000)>8)|((x \& 0 x f f 00)<<8)|((x \& 0 x f f)<<24)$. Other implementations rely on compiler built-in functions, or assembly code, possibly using dedicated processor instructions. Examples can be seen in the Linux implementations of the POSIX htons and htonl functions, converting values between host and network byte-order. Our analysis is able to analyze all the above $\mathrm{C} \mathrm{im-}$ plementations successfully, as well as alternative implementations (with stubs for assembly code). In the following of the paper, unless otherwise stated, we will implicitly refer to a version of Example 1 where variables have type uint16_t.

Approach. Low-level programs exhibit different semantics when run on platforms with different endiannesses. We thus model them as so-called double programs. The little-endian program is called the first (or left, or little-endian) version of the double program, while the big-endian program is called the second (or right, or big-endian) version. Both versions may share the same source code, or present syntactic differences (if conditional inclusion is used). Our approach to endian portability is to devise a joint, whole-program static analysis of a double program able to infer equivalences between the input-output relations of its versions. To this aim, we define a memory model able to represent a joint abstraction of their memories. We first parameterize a standard memory domain for low-level C programs with an explicit endianness parameter. Then, we lift it to double programs, and tailor it to infer, and represent symbolically, relevant equalities between little- and big-endian memories. We rely on a dedicated numerical domain based on symbolic predicates, to infer complementarity relations between individual bytes of program variables, such as those established by bitwise arithmetic operations. We validate our approach by analyzing large industrial low-level embedded C programs designed to be endian portable.

Related work. Several approaches to endian portability are developed in the literature. [31] relies on a source-to-source translation, which is only sound with respect to annotations provided by the programmer, whereas we require no annotations. [6] extends a compiler to generate code that executes with the opposite byte order semantics as the underlying architecture, at the cost of a performance penalty. Annotations are also required for soundness in some cases. [22] relies on dynamic analysis, which can find portability errors, but cannot prove endian portability formally, unlike our method. The SPARSE [7] static analysis tool used by Linux kernel developers relies on pervasive type annotations to detect endiannesses issues, but comes with no formal guarantee.

To our knowledge, no prior work uses sound static analysis to infer endian portability. Yet, our approach leverages prior work. We build on a memory abstract domain [25],[28, Sect. 5.2] developed for run-time error analysis of low-level $\mathrm{C}$ programs able to expose endian-dependent behaviors, and on double program 
semantics developed for patch analysis $[15,16]$. Our symbolic predicate domain is based on previous work on predicate domains [27], and symbolic constant propagation [26]. Our domain is also reminiscent of the Slice domain introduced in $[9,8]$ for another purpose, and implemented differently.

Contributions. The main contributions of this work are:

- We present a novel concrete collecting semantics, relating the behaviors of two versions of a program, running on platforms with different endiannesses.

- We propose a joint memory abstraction able to infer equivalence relations between little- and big-endian memories.

- We introduce a novel symbolic predicate domain to infer relations between individual bytes of the variables in the two programs, which has near-linear cost, and the right amount of relationality to express (bitwise) arithmetic properties relevant to endian portability.

- We implemented our analysis on the Mopsa [30,21] platform. Our prototype is able to scale to large real-world industrial software, with zero false alarms.

The paper is organised as follows. Section 2 formalizes the concrete collecting semantics, Sect. 3 describes the memory abstraction, Sect. 4 describes the numerical abstraction and introduces a novel numeric domain, Sect. 5 presents experimental results with a prototype implementation. Section 6 concludes.

\section{Syntax and concrete semantics}

Following the standard approach to abstract interpretation [11], we develop a concrete collecting semantics for a C-like language for double programs. The \| operator may occur anywhere in the parse tree to denote syntactic differences between the left (little-endian) and right (big-endian) versions of a double program. However, $\|$ operators cannot be nested: a double program only describes a pair of programs. Given double program $P$ with variables in $\mathcal{V}$, we call its left (resp. right) version $P_{1}=\pi_{1}(P)$ (resp. $P_{2}=\pi_{2}(P)$ ), where $\pi_{1}$ (resp. $\left.\pi_{2}\right)$ is a version extraction operator, defined by induction on the syntax, keeping only the left (resp. right) side of $\|$ symbols. For instance, $\pi_{1}(x \leftarrow 1 \| y \leftarrow 0)=x \leftarrow 1$, and $\pi_{2}(x \leftarrow 1 \| y \leftarrow 0)=y \leftarrow 0$, while $\pi_{1}(z \leftarrow 0)=z \leftarrow 0=\pi_{2}(z \leftarrow 0)$. Recall that syntactic differences between $P_{1}$ and $P_{2}$ may be distinct from semantic differences. Syntactically different statements may exhibit the same semantics in $P_{1}$ and $P_{2}$, like in Example 1, while syntactically equal statements may exhibit different semantics, like with the $\mathrm{C}$ statement $*((\operatorname{char} *) \& \mathrm{x})=1$, when integer variable $\mathrm{x}$ is such that $\operatorname{sizeof}(x)>1$.

\subsection{Syntax}

Simple programs $P_{1}$ and $P_{2}$ enjoy a standard, C-like syntax presented in Fig. 1. Statements stat are built on top of expressions expr and Boolean conditions cond. The syntax of double statements dstat includes specific assume_sync and assert_sync statements, used for specifications. The former is used to express 


$$
\begin{gathered}
\mathbb{E} \llbracket *_{\tau} e \rrbracket_{\alpha} \rho \triangleq\left\{v \mid\langle V, o\rangle \in \mathbb{E} \llbracket e \rrbracket_{\alpha} \rho \wedge 0 \leq o \leq \operatorname{sizeof}(V)-\operatorname{sizeof}(\tau)\right. \\
\left.\wedge v \in \operatorname{bec}_{\tau, \alpha}(\rho\langle V, o\rangle, \ldots, \rho\langle V, o+\operatorname{sizeof}(\tau)-1\rangle)\right\} \\
\mathbb{S} \llbracket *_{\tau} e_{1} \leftarrow e_{2} \rrbracket_{\alpha} R \triangleq \\
\bigcup_{\rho \in R}\left\{\rho\left[\forall i<\operatorname{sizeof}(\tau):\langle V, o+i\rangle \mapsto b_{i}\right] \mid\langle V, o\rangle \in \mathbb{E} \llbracket e_{1} \rrbracket_{\alpha} \rho\right. \\
\left.\wedge 0 \leq o \leq \operatorname{sizeof}(V)-\operatorname{sizeof}(\tau) \wedge\left(b_{0}, \ldots, b_{\text {sizeof }(\tau)-1}\right) \in \operatorname{benc}_{\tau, \alpha}\left(\mathbb{E} \llbracket e_{2} \rrbracket_{\alpha} \rho\right)\right\}
\end{gathered}
$$

Fig. 2. Concrete semantics of memory reads and writes.

integers) or pointer values. We denote the set of values as $\mathbb{\Xi} \triangleq \mathbb{Z} \cup \mathcal{P} t r$. The definition of the most concrete semantics requires a family of representation functions benc $_{\tau, \alpha} \in \mathbb{V} \rightarrow \mathcal{P}\left(\mathbb{B}^{*}\right)$, that convert a scalar value of given type $\tau \in$ scalar-type and endianness $\alpha \in \mathcal{A}$ into a sequence of $\operatorname{sizeof}(t)$ byte values. We denote as $\operatorname{bdec}_{\tau, \alpha} \in \mathbb{B}^{*} \rightarrow \mathcal{P}(\mathbb{V})$ the converse operation. For instance, on a 32-bit platform, benc $c_{\text {unsigned int, } \mathcal{L}}(1)=\{(1,0,0,0)\}, b d e c_{\text {unsigned short }, \mathcal{B}}(0,1)=\{1\}$, and benc $_{\mathbf{p t r}, \mathcal{L}}(p)=\{(\langle p, 0\rangle,\langle p, 1\rangle,\langle p, 2\rangle,\langle p, 3\rangle)\}$. This seemingly trivial encoding allows modeling copying pointer values byte per byte, as done e.g. by memcpy. Note that the benc $_{\tau, \alpha}$ and $b d e c_{\tau, \alpha}$ functions return a set of possible values. For instance, reinterpreting a pointer value as an integer, as in $b d e c_{\mathbf{i n t}, \mathcal{L}} \circ b e n c_{\mathbf{p t r}, \mathcal{L}}(p)$, returns the full range of type int. We do not detail the definitions of these functions here, for the sake of conciseness. An example may be found in [28, Sec. 5.2].

Environments are elements of $\mathcal{E} \triangleq \mathcal{A} d d r \rightarrow \mathbb{B}$. The semantics $\mathbb{E} \llbracket$ expr $\rrbracket \in$ $\mathcal{A} \rightarrow \mathcal{E} \rightarrow \mathcal{P}(\mathbb{V})$ and $\mathbb{S} \llbracket$ stat $\rrbracket \in \mathcal{A} \rightarrow \mathcal{P}(\mathcal{E}) \rightarrow \mathcal{P}(\mathcal{E})$ for simple expressions and statements is defined by standard induction on the syntax. We therefore only show, on Fig. 2, the semantics $\mathbb{E} \llbracket *_{\tau} e \rrbracket_{\alpha}$ and $\mathbb{S} \llbracket *_{\tau} e_{1} \leftarrow e_{2} \rrbracket_{\alpha}$ for memory reads and writes, given endianness $\alpha \in \mathcal{A}$. Bytes are fetched and decoded with $b d e c_{\tau, \alpha}$ when reading from memory in expression $*_{\tau} e$, while values computed by expression $e_{2}$ are encoded into bytes with benc $c_{\tau, \alpha}$ when writing to memory in assignment $*_{\tau} e_{1} \leftarrow e_{2}$. Note that illegal memory accesses are silently omitted to simplify the presentation.

\subsection{Semantics of double programs}

We now lift simple program semantics $\mathbb{S}$ to double program semantics $\mathbb{D}$. As both simple program versions $P_{k}=\pi_{k}(P)$ have concrete states in $\mathcal{E}$, the double program $P$ has concrete states in $\mathcal{D} \triangleq \mathcal{E} \times \mathcal{E}$. The semantics of $P_{k}$ is parameterized by its endianness $\alpha_{k} \in \mathcal{A}$. We assume, without loss of generality, that $P_{1}$ is the little-endian version, and $P_{2}$ the big-endian one.

$\mathbb{D} \llbracket s \rrbracket \in \mathcal{P}(\mathcal{D}) \rightarrow \mathcal{P}(\mathcal{D})$ describes the relation between input and output states of $s$, which are pairs of states of simple programs. The definition for $\mathbb{D} \llbracket s \rrbracket$ is shown on Fig. 3. $\mathbb{D}$ leverages previous work on patch analysis $[15,16]$. It is defined by induction on the syntax, so as to allow for a modular definition and joint analyses of double programs. Note that $\mathbb{D}$ is parametric in $\mathbb{S}$.

The semantics for the empty program is the identity function. The semantics $\mathbb{D} \llbracket s_{1} \| s_{2} \rrbracket$ for the composition of two syntactically different statements reverts 


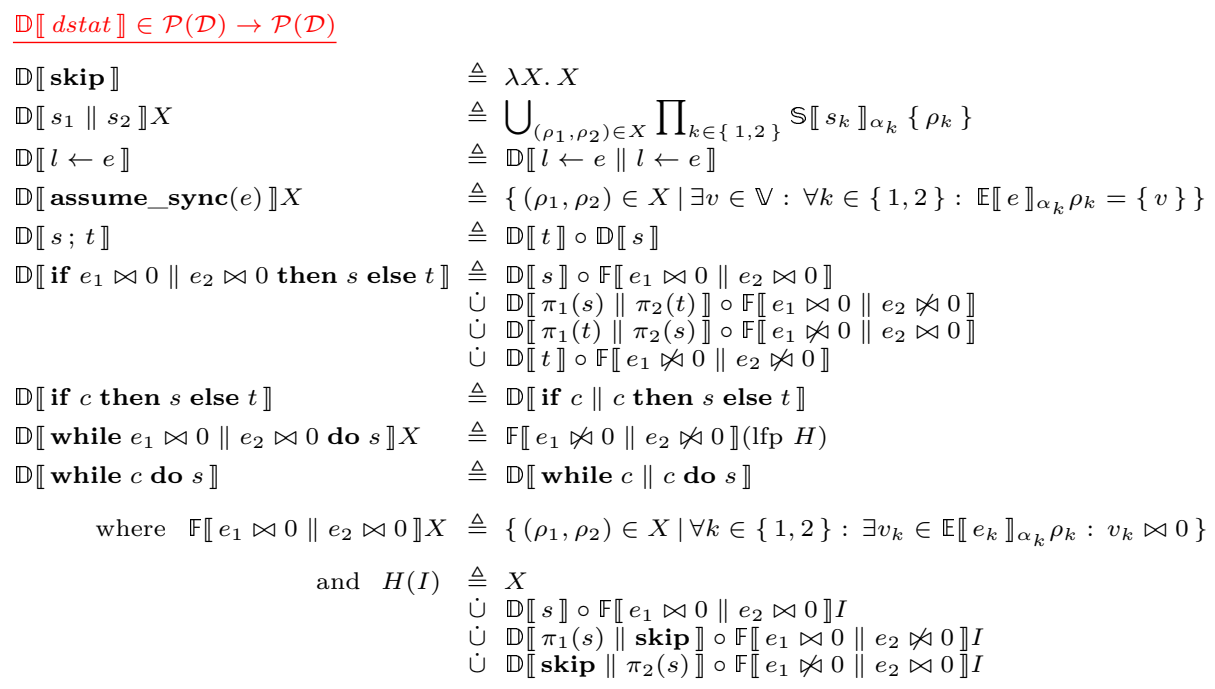

Fig. 3. Denotational concrete semantics of double programs.

to the pairing of the simple program semantics of individual simple statements $s_{1}$ and $s_{2}$. The semantics for assignments is defined with this construct. The semantics of assume_sync and assert_sync statements filters away environments where the left and right versions of a double program may disagree on the value of expression $e$. In addition, assert_sync raises an alarm if $e$ may evaluate to different values in $P_{1}$ and $P_{2}$. We omit alarms from the semantics for conciseness. The semantics for the sequential composition of statements boils down to the composition of the semantics of individual statements. The semantics for selection statements relies on the filter $\mathbb{F} \llbracket e_{1} \bowtie 0 \| e_{2} \bowtie 0 \rrbracket$ to distinguish between cases where both versions agree on the value of the controlling expression, and cases where they do not (a.k.a. unstable tests). There are two stable and two unstable test cases, according to the evaluations of the two conditions. The semantics for stable test cases is standard. The semantics for unstable test cases is defined by the composition of left version of the then branch, filtered by the condition, and of the right version of the else branch, filtered by the negation of the condition (and the dual case). The semantics for (possibly unbounded) iteration statements is defined using the least fixpoint of a function defined similarly.

\subsection{Properties of Interest}

We wish to prove the functional equivalence between the left and right versions of a given double program $P \in d s t a t$, restricted to a set of distinguished outputs, specified with the assert_sync primitive. Let $x_{0} \in \mathcal{D}$ be an initial doubleprogram state. The set of states reachable by $P$ is $\mathbb{D} \llbracket P \rrbracket\left\{x_{0}\right\}$. Let $\Omega$ be a set of output left-values of program $P$. The property of interest is that $\pi_{1}(P)$ and 


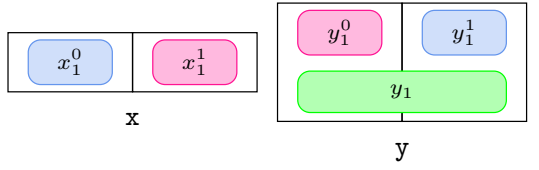

(a) Program 1

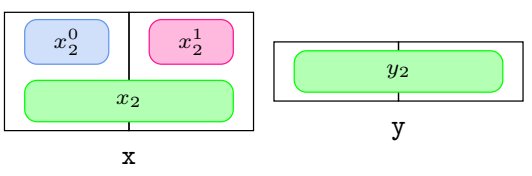

(b) Program 2

Fig. 4. Memory cells of Example 1: $\square=b_{0}, \square=b_{1}, \square=b_{0} \times 2^{8}+b_{1}$.

$\pi_{2}(P)$ compute equal values for all outputs:

$$
\forall l \in \Omega: \forall\left\langle\rho_{1}, \rho_{2}\right\rangle \in \mathbb{D} \llbracket P \rrbracket\left\{x_{0}\right\}: \exists v \in \mathbb{V}: \forall k \in\{1,2\}: \mathbb{E} \llbracket l \rrbracket_{\alpha_{k}} \rho_{k}=\{v\} .
$$

For instance, let $\mathrm{S}$ denote the set of reachable states of Example 1, before line 8: $S=\left\{\left\langle\left[x_{1}^{o} \mapsto b_{o}, y_{1}^{o} \mapsto b_{1-o}\right],\left[x_{2}^{o} \mapsto b_{o}, y_{2}^{o} \mapsto b_{o}\right]\right\rangle \mid o \in\{0,1\},\left(b_{0}, b_{1}\right) \in[0,255]^{2}\right\}$, where $b_{0}$ and $b_{1}$ denote the values of bytes read from the network, and we write $x_{i}^{o}$ and $y_{i}^{o}$ for $\langle x, o\rangle$ and $\langle y, o\rangle$ in program version $i$. The portability property expressed at line 8 is $y_{1}^{0}+2^{8} y_{1}^{1}=2^{8} y_{2}^{0}+y_{2}^{1}$, which can be proved from $S$.

Our concrete collecting semantics $\mathbb{D}$ is not computable in general. We will thus rely on computable abstractions, to infer this property by static analysis. Note that the use of assume_sync and assert_sync in specifications allows for both whole-program analysis, and separate analyses of program parts.

\section{Memory abstraction}

Though we aim at designing a computable abstract semantics in Sect. 4, we first tailor a (non computable) abstraction of our memory model. We rely on the Cells memory abstraction of simple programs [25],[28, Sect. 5.2]. In order to handle $\mathrm{C}$ programs computing with machine integers of multiple sizes, with bytelevel access to their encoding through type-punning, this domain represents the memory as a dynamic collection of scalar variables, termed cells, holding values for the scalar memory dereferences discovered during the analysis. It maintains a consistent abstract state despite the introduction of overlapping cells by typepunning. We lift this memory abstraction to double programs, and we extend it for representing equalities between cells symbolically.

\section{$3.1 \quad$ Cells}

We first consider the finite universe Cell $\triangleq \mathcal{V} \times \mathbb{N} \times$ scalar-type $\times \mathcal{A}$ of cells of one program. A cell $\langle V, o, \tau, \alpha\rangle \in \mathrm{Cell}$ is denoted as a variable $V$, an offset $o$, and information specifying the encoding of values: a scalar type $\tau$ and endianness $\alpha$. To account for both programs, we introduce projected cells as $\widetilde{\mathcal{C e l l}} \triangleq \mathrm{Cell} \times\{1,2\}$, where 1 (resp. 2) denotes a cell in the memory of $P_{1}$ (resp. $P_{2}$ ).

For instance, consider the program in Example 1. We show in Fig. 4 the cells synthesized at the end of the program. Let $x_{k} \triangleq\left\langle x, 0, \mathbf{u 1 6}, \alpha_{k}, k\right\rangle$ denote 2 -byte 
cells for $\mathrm{x}$ in Program $k \in\{1,2\}$, where $\alpha_{1}=\mathcal{L}$ and $\alpha_{2}=\mathcal{B}$. 1-byte cells are denoted as $x_{k}^{o} \triangleq\left\langle x, o, \mathbf{u} 8, \alpha_{k}, k\right\rangle$ where $o \in\{0,1\}$. The cells for y are defined in a similar way. Both program versions first call function read_from_network, which reads a stream of bytes from an external source, and writes it into a buffer. The same stream is read by both program versions. A stub for read_from_network is shown in Fig. 5. After completion of the call, we have $x_{1}^{0}=b_{0}=x_{2}^{0}$ and

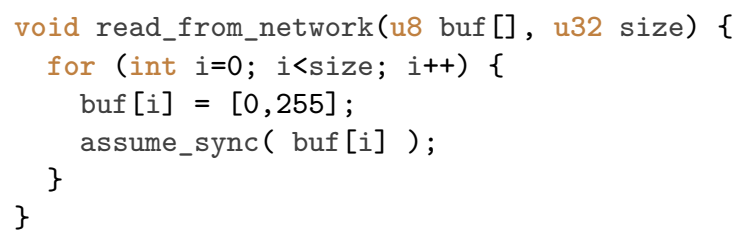

Fig. 5. Stub for read_from_network function.

$x_{1}^{1}=b_{1}=x_{2}^{1}$, where $b_{0}$ and $b_{1}$ are the first and second bytes read from the network, respectively. Then, Program 1 swaps the bytes of $\mathrm{x}$ into those of $\mathrm{y}$ : $x_{1}^{0}=y_{1}^{1}$ and $x_{1}^{1}=y_{1}^{0}$. Program 2, in contrast, assigns $\mathrm{x}$ to $\mathrm{y} . \mathrm{x}$ is thus read as a 2-byte cell, while only 1-byte cells are present. Therefore, the Cells domain synthesizes $x_{2}$ by adding the constraint $x_{2}=2^{8} x_{2}^{0}+x_{2}^{1}$, following big-endian byte-order, before performing the assignment $y_{2} \leftarrow x_{2}$. To sum up, we obtain the following constraints:

$$
x_{1}^{0}=x_{2}^{0}=y_{1}^{1} \quad x_{1}^{1}=x_{2}^{1}=y_{1}^{0} \quad y_{2}=x_{2}
$$

In addition to the cell constraints on $\mathrm{x}$ and $\mathrm{y}$ :

$$
x_{1}=x_{1}^{0}+2^{8} x_{1}^{1} \quad y_{1}=y_{1}^{0}+2^{8} y_{1}^{1} \quad x_{2}=2^{8} x_{2}^{0}+x_{2}^{1} \quad y_{2}=2^{8} y_{2}^{0}+y_{2}^{1}
$$

Our goal is to prove that $y_{1}=y_{2}$ given such constraints. To do so, we want to leverage numerical domains to abstract the values of cells. However, such constraints require an expressive domain, such as polyhedra or linear equalities, that can hamper the scalability of the analysis. In addition, we note that we need to infer many equalities, most of them between the left and right versions of the same cells. This is no surprise as we expect most variables to hold equal values in the little- and big-endian memories most of the time, with only local differences. Rather than relying completely on the expressiveness of the underlying numeric domain, we first optimize our memory model for this common case, introducing the concept of shared bi-cells, which act as a symbolic representation of cells equality.

\subsection{Shared bi-cells}

We denote as $\mathcal{B}$ icell $\triangleq \widetilde{\text { Cell }} \cup(\widetilde{\text { Cell }} \times \widetilde{\text { Cell }})$ the set of bi-cells. A bi-cell is either a projected cell in $\widetilde{\mathcal{C e l l}}$, or a pair of such cells in $\widetilde{\mathrm{Cell}} \times \widetilde{\mathrm{Cell}}$ assumed to hold equal 
value, called a shared bi-cell. Bi-cell sharing allows a single representation, in the memory environment, for two projected cells from different program versions at the same memory location and holding equal values. Abstract memory states of double programs are modeled as a choice of a set of bi-cells $C \subseteq \mathcal{B}$ icell, and a set of scalar environments on $C$. Let $\mathcal{D}^{b} \triangleq \bigcup_{C \subseteq \mathcal{B} \text { icell }}\{\langle C, R\rangle \mid R \in \mathcal{P}(C \rightarrow \mathbb{V})\}$ be the associated abstract domain. An abstract state represents a set of concrete byte-level memories in $\mathcal{D}=\mathcal{E} \times \mathcal{E}$. The values of the bytes of these memories must satisfy all the numeric constraints on bi-cells implied by the environments: $\gamma_{\mathcal{B} i c e l l}\langle C, R\rangle \triangleq\left\{\left(\mu_{1}, \mu_{2}\right) \in \mathcal{D} \mid \exists \rho \in R: \forall c_{k}=\langle V, o, \tau, \alpha, k\rangle \in \widetilde{\mathcal{C e l l}}:\right.$ $\forall c \in$ occ $\left(c_{k}, C\right): \exists\left(b_{0}, \ldots, b_{\text {sizeof }(\tau)-1}\right) \in$ benc $_{\tau, \alpha}(\rho(c)):$ $\left.\forall 0 \leq i<\operatorname{sizeof}(\tau): \mu_{k}\langle V, o+i\rangle=b_{i}\right\}$

where occ $\in \widetilde{\mathrm{Cell}} \times \mathcal{P}($ Bicell $) \rightarrow \mathcal{P}(\mathcal{B}$ icell $)$ records occurrences of a projected cell among bi-cells: occ $(c, C) \triangleq\left\{c^{\prime} \in C \mid c^{\prime}=c \vee \exists c^{\prime \prime}: c^{\prime}=\left\langle c, c^{\prime \prime}\right\rangle \vee c^{\prime}=\left\langle c^{\prime \prime}, c\right\rangle\right\}$.

In Fig. 6, we depict the bi-cells obtained after analyzing the program shown

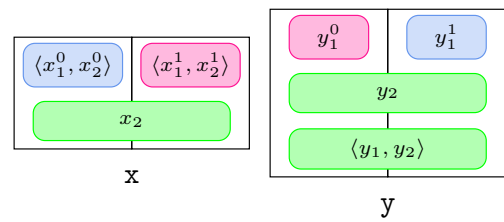

Fig. 6. Shared bi-cells of Example 1. in Example 1. For variable $\mathrm{x}$, since read_from_network writes the same value to $x_{1}^{0}$ and $x_{2}^{0}$, we can synthesize the shared bi-cell $\left\langle x_{1}^{0}, x_{2}^{0}\right\rangle$ to represent the equality $x_{1}^{0}=x_{2}^{0}$. In a similar way, we synthesize the shared bi-cell $\left\langle x_{1}^{1}, x_{2}^{1}\right\rangle$. Therefore, as opposed to the separate representation of the memories of Programs 1 and

2 in Fig. 4, the joint representation induced by bi-cell sharing allows reducing the burden on numeric domains. In the following, we describe more involved cell synthesis operations that allow us to realize $\left\langle y_{1}, y_{2}\right\rangle$, and thus to infer that $y_{1}=y_{2}$.

\subsection{Cell synthesis}

A cornerstone of our memory model is bi-cell synthesis. In order to read or write a scalar value to a given location of memory, we must create a suitable bi-cell, or retrieve an existing one from the environment. To guarantee the soundness of the analysis when adding a new bi-cell, it is necessary to ensure that values assigned to it are consistent with those of existing overlapping bi-cells. Our memory domain first attempts to synthesize shared bi-cells if an equality can be inferred from the environment, by pattern-matching. In case of failure, it safely defaults to a pair of projected bi-cells, the values of which are set according to those of existing overlapping bi-cells.

We have already used shared bi-cell synthesis implicitly on Fig. 6. When reading variable $\mathrm{y}$ at the end of Example 1, the memory domain attempts to synthesize $\left\langle y_{1}, y_{2}\right\rangle$, as a proof of $y_{1}=y_{2}$. To this aim, it searches, among possible patterns, for an existing cell, equal to both $y_{1}$ and $y_{2} . x_{2}$ is a candidate, assuming equality $x_{2}=y_{2}$ is recorded in (an abstraction of) the environment. Then the domain looks for 1-byte bi-cells for $y_{1}$ and $x_{2}$, and finds the four blue and red 


$$
\begin{gathered}
\phi^{b}\langle V, o, \tau\rangle\langle C, R\rangle \triangleq \quad \text { if } \operatorname{equal}\left(c_{1}, c_{2}, \alpha_{1}, \alpha_{2}\right)\langle C, R\rangle \\
\left\{\begin{array}{lr}
\left\langle c_{1}, c_{2}\right\rangle & \text { else if } \operatorname{equal}\left(c_{1}, c_{2}, \alpha_{2}, \alpha_{2}\right)\langle C, R\rangle \\
\left\langle c_{1}^{*}, c_{2}\right\rangle \quad \text { else if } \operatorname{equal}\left(c_{1}, c_{2}, \alpha_{1}, \alpha_{1}\right)\langle C, R\rangle \\
\left\langle c_{1}, c_{2}^{*}\right\rangle \quad \text { otherwise }
\end{array}\right.
\end{gathered}
$$

$$
\text { where } \begin{aligned}
c_{i} & =\left\langle V, o, \tau, \alpha_{i}, i\right\rangle, \\
c_{i}^{*} & =\left\langle V, o, \tau, \alpha_{3-i}, i\right\rangle, \\
\alpha_{1} & =\mathcal{L}, \text { and } \\
\alpha_{2} & =\mathcal{B} .
\end{aligned}
$$

Fig. 7. Shared bi-cell synthesize function.

cells from Fig. 6. As $y_{1}$ and $x_{2}$ have opposite endian encodings, it queries the environment for equalities $y_{1}^{0}=\left\langle x_{1}^{1}, x_{2}^{1}\right\rangle$ and $y_{1}^{1}=\left\langle x_{1}^{0}, x_{2}^{0}\right\rangle$. The success of the synthesis relies on pattern-matching, and three equalities which may be inferred by a numerical domain implementing simple symbolic propagation.

Shared bi-cell synthesis. More generally, function $\phi^{b}$ formalizes the patterns matched attempting to synthesize a shared bi-cell for a given dereference $c \in \mathcal{C e l l}_{0} \triangleq \mathcal{V} \times \mathbb{N} \times$ scalar-type. An implementation is proposed in Fig. 7 . Firstly, it returns $\left\langle c_{1}, c_{2}\right\rangle$ if $c_{1}=c_{2}$ may be inferred from the environment, where $c_{i}=\left\langle c, \alpha_{i}, i\right\rangle$ are projected versions of $c$, with the native endiannesses of their respective platforms. Otherwise, it returns $\left\langle c_{1}^{*}, c_{2}\right\rangle$, where $c_{1}^{*}$ is a big-endian projected bi-cell of Program 1, if $c_{1}^{*}=c_{2}$ holds. For instance, $\left\langle x_{1}^{*}, x_{2}\right\rangle$ will be synthesized if variable $\mathrm{x}$ is read after the end of Example 1. Otherwise, it returns $\left\langle c_{1}, c_{2}^{*}\right\rangle$, where $c_{2}^{*}$ is a little-endian projected bi-cell of Program 2, if $c_{1}=c_{2}^{*}$ holds. Finally, if all fails, it returns an error $\top . \phi^{b} \in \mathcal{C e l l}_{0} \rightarrow \mathcal{D}^{b} \rightarrow \widetilde{\mathcal{C e l l}}^{2} \cup\{\top\}$ relies on predicate equal to compare two projected bi-cells of the same type, with specified endianness encodings. An implementation is shown on Fig. 8. equal returns true when compared cells are part of a shared bi-cell, or when equality is ensured by the environment. Otherwise, it compares individual 1-byte bi-cells of the same weights $2^{8 w}$, at endianness-dependent offsets: $\operatorname{offset}(w, s, \alpha) \triangleq w$ if $\alpha=\mathcal{L}$, and $s-w-1$ otherwise. Otherwise, equal searches for candidate projected bi-cells in the environment, equal to both $c$ and $c^{\prime}$. In the formula, we denote the set of projected bi-cells in the environment as flatten $(C) \triangleq\left\{c \in \widetilde{\mathcal{C e l l}} \mid c \in C \vee \exists c^{\prime} \in C\right.$ : $\left.\left\langle c, c^{\prime}\right\rangle \in C \vee\left\langle c^{\prime}, c\right\rangle \in C\right\}$. equal returns true in case of success, false otherwise.

Projected bi-cell synthesis. If all attempts to synthesize a shared bi-cell $\left\langle c_{1}, c_{2}\right\rangle,\left\langle c_{1}^{*}, c_{2}\right\rangle$, or $\left\langle c_{1}, c_{2}^{*}\right\rangle$ fail, our memory domain creates the pair of projected bi-cells $c_{1}$ and $c_{2}$ instead. To set their values soundly, it calls $\phi_{1}\left(c_{1}\right)(C)$ and $\phi_{2}\left(c_{2}\right)(C)$, where $\phi_{i}\left(c_{i}\right)(C)$ returns a syntactic expression denoting (an abstraction of) the value of $c_{i}$ as a function of cells existing in $C$. For instance, $\phi_{1}(\langle y, 0, \mathbf{u 1 6}, \mathcal{B}, 1\rangle)(C)=2^{8} y_{1}^{0}+y_{1}^{1}$ at the end of Example 1 (see Fig. 6).

To define the synthesize functions $\phi_{1}$ and $\phi_{2} \in \widetilde{\mathcal{C e l l}} \rightarrow \mathcal{P}(\mathcal{B i c e l l}) \rightarrow$ expr for projected bi-cells, we first need to define a generic cell synthesize function $\phi \in \mathrm{Cell} \rightarrow \mathcal{P}(\mathrm{Cell}) \rightarrow$ expr , such that $\phi(c)(C)$ returns a syntactic expression 


$$
\begin{aligned}
& \text { equal }\left(\langle V, o, \tau,-, k\rangle,\left\langle V^{\prime}, o^{\prime}, \tau,-, k^{\prime}\right\rangle, \alpha, \alpha^{\prime}\right)\langle C, R\rangle \triangleq \\
& \quad \text { let } c=\langle V, o, \tau, \alpha, k\rangle \text { and } c^{\prime}=\left\langle V^{\prime}, o^{\prime}, \tau, \alpha^{\prime}, k^{\prime}\right\rangle \text { and } s=\operatorname{sizeof}(\tau) \text { in } \\
& \quad\left\langle c, c^{\prime}\right\rangle \in C \vee\left\langle c^{\prime}, c\right\rangle \in C \vee \\
& \quad\left(\exists\left(x, x^{\prime}\right) \in \operatorname{occ}(c, C) \times \operatorname{occ}\left(c^{\prime}, C\right): \forall \rho \in R: \rho(x)=\rho\left(x^{\prime}\right)\right) \vee \\
& \quad\left(\forall 0 \leq w<s: \text { equal }\left(c^{\text {offset }(w, s, \alpha)}, c^{\prime} \operatorname{offset}\left(w, s, \alpha^{\prime}\right), \alpha, \alpha^{\prime}\right)\langle C, R\rangle\right) \vee \\
& \quad\left(\exists x \in \operatorname{flatten}(C) \backslash\left\{c, c^{\prime}\right\}: \operatorname{equal}\left(c, x, \alpha, \alpha_{x}\right)\langle C, R\rangle \wedge \operatorname{equal}\left(c^{\prime}, x, \alpha^{\prime}, \alpha_{x}\right)\langle C, R\rangle\right)
\end{aligned}
$$

where $c^{p}$ denotes the 1-byte bi-cell $\langle V, o+p, \mathbf{u} \mathbf{8}, \alpha, k\rangle$ (and respectively for $c^{\prime p}$ ), and $\alpha_{x}$ denotes the endianness encoding of $x$.

Fig. 8. Equality test between projected bi-cells.

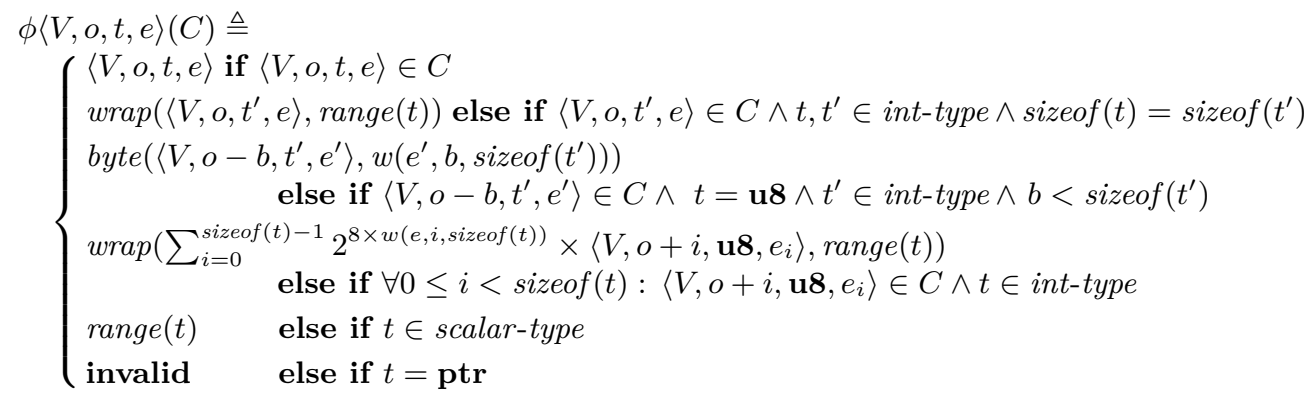

Fig. 9. Generic cell synthesize function.

denoting (an abstraction of) the value of the cell $c$ as a function of cells in $C . \phi$ is designed as an extension to multiple endianness encodings of the cell synthesize function originally proposed in [28, sec. 5.2].

An example implementation is proposed in Fig. 9. Firstly, if the cell already exists $(c \in C)$, it is directly returned by $\phi$. Otherwise, $\phi$ looks for integer cells of the same size and different signedness, and converts them using function wrap to model wrap-around, and function range for the range of the type: $\operatorname{wrap}(v,[l, h]) \triangleq$ $\min \left\{v^{\prime} \mid v^{\prime} \geq l \wedge \exists k \in \mathbb{Z}: v=v^{\prime}+k(h-l+1)\right\}$, and $\operatorname{range}(t) \triangleq\left[0,2^{8 s}-1\right]$ if $t$ is unsigned, and $\left[-2^{8 s-1}, 2^{8 s-1}-1\right]$ if $t$ is signed, where $s=$ sizeof $(t)$. Thirdly, $\phi$ extracts unsigned bytes from integers. Fourthly, $\phi$ aggregates unsigned bytes into integers. Function $w \in \mathcal{A} \times \mathbb{N}^{2} \rightarrow \mathbb{N}$ is used to model the endianness-dependent weight of bytes in integers: $w(\mathcal{L}, b, s) \triangleq b$ and $w(\mathcal{B}, b, s) \triangleq s-b-1$. The value of the byte of weight $2^{8 w}$ in integer $x$ is: byte $(x, w)=\left\lfloor x / 2^{8 w}\right\rfloor \bmod 2^{8}$. When all fails, $\phi$ returns the full range of the type (or invalid, for a pointer). Many definitions are possible for $\phi$, e.g. adding cases to support floats, or to synthesize integer cells from cells of opposite endianness.

To define $\phi_{1}$ and $\phi_{2}$, we project bi-cells of the appropriate side onto cells, apply $\phi$, and lift the resulting cell expression back to a bi-cell expression. More precisely, to compute $\phi_{1}\langle c, 1\rangle(C)$, we first project the bi-cell set $C$ to the cells of program version $1: C_{1} \triangleq\{x \mid\langle x, 1\rangle \in C \vee \exists y:\langle\langle x, 1\rangle,\langle y, 2\rangle\rangle \in C\}$. 
Then, we retrieve the constraints on cell $c$ by applying the generic cell synthesize function: $e_{1} \triangleq \phi(c)\left(C_{1}\right)$. Finally, $\phi_{1}\langle c, 1\rangle(C)$ is obtained by substituting every cell $x$ occurring in $e_{1}$ with an element of $o c c(\langle x, 1\rangle, C)$. Note that $e_{1}$ is a syntactic expression over cells in $C_{1}$, and $\operatorname{occ}(\langle x, 1\rangle, C) \neq \emptyset$ for all $x \in C_{1}$. The definition of $\phi_{2}\langle c, 2\rangle(C)$ is analogue.

Cell addition. Cell addition, add-cell Cell $_{0} \rightarrow \mathcal{D}^{b} \rightarrow \mathcal{D}^{b}$, then simply adds the cell(s) and initializes their value(s).

$$
\begin{aligned}
& \text { add-cell }(c)\langle C, R\rangle \triangleq \\
& \quad \text { if } \phi^{b}(c)\langle C, R\rangle=\left\langle x_{1}, x_{2}\right\rangle \text { then } \\
& \left\langle C \cup\left\{\left\langle x_{1}, x_{2}\right\rangle\right\},\left\{\rho\left[\left\langle x_{1}, x_{2}\right\rangle \mapsto v\right] \mid \rho \in R, v \in \mathbb{E} \llbracket \phi_{1}\left(x_{1}\right)(C) \rrbracket \alpha_{\alpha_{1}} \rho\right\}\right\rangle \\
& \text { else } \\
& \quad\left\langle C \cup\left\{c_{1}, c_{2}\right\},\left\{\rho\left[\forall i: c_{i} \mapsto v_{i}\right] \mid \rho \in R, \forall i: v_{i} \in \mathbb{E} \llbracket \phi_{i}\left(c_{i}\right)(C) \rrbracket \alpha_{\alpha_{i}} \rho\right\}\right\rangle
\end{aligned}
$$

where $c_{1}=\langle c, \mathcal{L}, 1\rangle$ and $c_{2}=\langle c, \mathcal{B}, 2\rangle$.

\subsection{Abstract join}

The abstract join must merge environment sets defined on heterogeneous bicell sets. We therefore define a unification function unify $\in\left(\mathcal{D}^{b}\right)^{2} \rightarrow\left(\mathcal{D}^{b}\right)^{2}$. unify $\left(\left\langle C_{1}, R_{1}\right\rangle,\left\langle C_{2}, R_{2}\right\rangle\right)$ adds, with add-cell ${ }^{b}$, any missing cells to $\left\langle C_{1}, R_{1}\right\rangle$ and $\left\langle C_{2}, R_{2}\right\rangle$ : respectively $C_{2} \backslash C_{1}$ and $C_{1} \backslash C_{2}$. Let $\left\langle C_{1}^{\prime}, R_{1}^{\prime}\right\rangle$ and $\left\langle C_{2}^{\prime}, R_{2}^{\prime}\right\rangle$ be the resulting abstract states. $C_{1}^{\prime}$ and $C_{2}^{\prime}$ may include both projected and shared bi-cells. A shared bi-cell that does not occur in both $C_{1}^{\prime}$ and $C_{2}^{\prime}$ cannot be soundly included in the unified state, as it conveys equality information that holds for one abstract state only. All such cells are thus removed before unification. Formally, unify $\left(\left\langle C_{1}, R_{1}\right\rangle,\left\langle C_{2}, R_{2}\right\rangle\right)=\left(\left\langle C_{12}, R_{1}^{\prime \prime}\right\rangle,\left\langle C_{12}, R_{2}^{\prime \prime}\right\rangle\right)$, where $C_{12}=$ $\left(C_{1}^{\prime} \cup C_{2}^{\prime}\right) \backslash\left(\left(\left(C_{1}^{\prime} \cup C_{2}^{\prime}\right) \backslash \widetilde{\mathcal{C e l l}}\right) \backslash\left(C_{1}^{\prime} \cap C_{2}^{\prime}\right)\right)$, and $R_{k}^{\prime \prime}=\left\{\rho_{\mid C_{12}} \mid \rho \in R_{k}^{\prime}\right\}$. The abstract join may now be defined as: $\left\langle C_{1}, R_{1}\right\rangle \sqcup\left\langle C_{2}, R_{2}\right\rangle \triangleq\left\langle C_{12}, R_{1}^{\prime \prime} \cup R_{2}^{\prime \prime}\right\rangle$.

\subsection{Semantics of simple statements}

Before defining the semantics for double statements in this domain, we first define the semantics $\mathbb{E}_{k}^{b} \llbracket *_{t} e \rrbracket \in \mathcal{D}^{b} \rightarrow \mathcal{P}(\mathbb{V})$ and $\mathbb{S}_{k}^{b} \llbracket *_{t} e_{1} \leftarrow e_{2} \rrbracket \in \mathcal{P}\left(\mathcal{D}^{b}\right) \rightarrow \mathcal{P}\left(\mathcal{D}^{b}\right)$ for simple memory reads and writes, in program version $k \in\{1,2\}$.

Evaluations. To compute $\mathbb{E}_{k}^{b} \llbracket *_{t} e \rrbracket\langle C, R\rangle$, we first resolve $*_{t} e$ into a set $L$ of projected bi-cells on side $k$, by evaluating $e$ into a set of pointer values, and gathering projected bi-cells corresponding to valid pointers:

$$
L \triangleq\left\{\left\langle V, o, t, \alpha_{k}, k\right\rangle \mid\langle V, o\rangle \in \mathbb{E} \llbracket e \rrbracket_{\alpha_{k}} \rho, \rho \in R, 0 \leq o \leq \operatorname{sizeof}(V)-\operatorname{sizeof}(t)\right\}
$$

Then, we call $a d d$-cell ${ }^{b}$ to ensure that all the target cells in $L$ are in the abstract environment, which updates $\langle C, R\rangle$ to $\left\langle C_{0}, R_{0}\right\rangle$. Finally: $\mathbb{E}_{k}^{b} \llbracket *_{t} e \rrbracket\langle C, R\rangle=$ $\left\{\rho(c) \mid \rho \in R_{0}, c \in L\right\}$. 
Assignments. The semantics of assignments $\mathbb{S}_{k}^{b} \llbracket *_{t} e_{1} \leftarrow e_{2} \rrbracket\langle C, R\rangle$ involves more steps. Like for evaluations, we start with resolving $*_{t} e_{1}$ into a set $L$ of projected bi-cells on side $k$. Then, we realize the cells in $L$ using $a d d$-cell $l^{b}$ : let $\left\langle C_{0}, R_{0}\right\rangle$ be the updated environment. Some of the projected bi-cells in $L$ may have been realized into shared bi-cells. Let $S \triangleq\left(C_{0} \backslash C\right) \cap \widetilde{\mathrm{Cell}}^{2}$ be the set of such shared bi-cells. Elements of $S$ represent equalities between bi-cells projected on side $k$, and on side opposite to $k$. Such equalities may no longer hold, after assignment on side $k$. Therefore, we split shared bi-cells of $S$ into their left and right projections, in a copy-on-write strategy. The updated environment is:

$\left\langle C_{0}^{\prime}, R_{0}^{\prime}\right\rangle=\left\langle C_{0} \cup \bigcup_{\left\langle c, c^{\prime}\right\rangle \in S}\left\{c, c^{\prime}\right\},\left\{c \mapsto\left\{\begin{array}{l}\rho(x) \text { if } \exists x \in \text { occ }(c, S) \neq \emptyset \\ \rho(c) \text { otherwise }\end{array} \mid \rho \in R_{0}\right\}\right\rangle\right.$

Finally, we update the environment for the projected bi-cells written (elements of $L$ ), with the possible values of $e_{2}$. However, this is not sufficient: it is also necessary to update the environment for any overlapping bi-cells, including shared bi-cells that have been split into pairs of projected cells. A sound and efficient (though possibly coarse) solution is to simply remove them. Indeed, removing any bi-cell is always sound in our memory model: it amounts to losing information, as we loose constraints on the byte-representation of the memory. Let $\Omega \subseteq \mathcal{C}_{0}^{\prime} \backslash L$ be the set of such bi-cells: elements of $\Omega$ are shared bi-cells and projected bi-cells on side $k$, with offsets and sizes such that they overlap some element of $L$. The updated environment is:

$$
\begin{aligned}
\mathbb{S}_{k}^{b} \llbracket *_{t} e_{1} \leftarrow e_{2} \rrbracket\langle C, R\rangle= & \left\langle C_{0}^{\prime} \backslash \Omega,\right. \\
& \left.\left\{\rho_{\mid C_{0}^{\prime} \backslash \Omega}[\forall c \in L: c \mapsto v] \mid \rho \in R_{0}^{\prime}, v \in \mathbb{E}_{k}^{b} \llbracket e_{2} \rrbracket\left\langle C_{0}^{\prime}, R_{0}^{\prime}\right\rangle\right\}\right\rangle
\end{aligned}
$$

\subsection{Semantics of double statements}

We are now ready to define the semantics $\mathbb{D}^{b} \llbracket d s t a t \rrbracket \in \mathcal{D}^{b} \rightarrow \mathcal{D}^{b}$ of double statements in this domain. Like $\mathbb{D}, \mathbb{D}^{b}$ is defined by induction on the syntax. We focus on base cases, as inductive cases are unchanged.

The semantics $\mathbb{D}^{b} \llbracket s_{1} \| s_{2} \rrbracket$ for two syntactically different statements composes simple programs semantics: $\mathbb{D}^{b} \llbracket s_{1} \| s_{2} \rrbracket \triangleq \mathbb{S}_{2}^{b} \llbracket s_{2} \rrbracket \circ \mathbb{S}_{1}^{b} \llbracket s_{1} \rrbracket$. The semantics for assume_sync, assert_sync, and $\mathbb{F}^{b} \llbracket e_{1} \bowtie 0 \| e_{2} \bowtie 0 \rrbracket$ are mostly unchanged, but for symbolic simplifications taking advantage of symbolic representations of equalities in our domain, for improved efficiency and precision. In particular, when $e$ is a deterministic expression containing a single dereference, then $\mathbb{D}^{b} \llbracket$ assume_sync $(e) \rrbracket$ adds a shared bi-cell for this dereference to the abstract environment. Consistently, $\mathbb{D}^{b} \llbracket$ assert_sync $(e) \rrbracket$ first tests whether $e$ is deterministic, and its dereferences evaluate to shared bi-cells. In this case, $\mathbb{D}^{b} \llbracket$ assert_sync $(e) \rrbracket$ raises no alarm. Otherwise, the semantics uses environment functions $\rho$ to test equalities of bi-cell values, like for $\mathbb{D}$. A similar symbolic simplification is used for the $\mathbb{F}^{b} \llbracket \cdot \rrbracket$ filter: $\mathbb{F}^{b} \llbracket e \bowtie 0 \| e \bowtie 0 \rrbracket\langle C, R\rangle=\emptyset$ (hence the test is stable) when $e$ is deterministic and all dereferences evaluate to shared bi-cells, which is the common case. For instance, when evaluating $\mathbb{D} \llbracket$ if $(x<y)$ then $s$ else $t \rrbracket$, if the dereferences for variable $\mathrm{x}$ and $\mathrm{y}$ evaluate to shared bi-cells, the two unstable tests cases are $\perp$. 
Assignments. In an assignment $\mathbb{D}^{b} \llbracket *_{t} e_{1} \leftarrow e_{2} \rrbracket\langle C, R\rangle$, although both programs execute the same syntactic assignment, their semantics are different, as are their endiannesses. In addition, available bi-cells may be different. By default, double assignments are straightforward extensions of simple assignments: $\mathbb{D}^{\mathrm{b}} \llbracket *_{t} e_{1} \leftarrow e_{2} \rrbracket=\mathbb{S}_{2}^{b} \llbracket *_{t} e_{1} \leftarrow e_{2} \rrbracket \circ \mathbb{S}_{1}^{b} \llbracket *_{t} e_{1} \leftarrow e_{2} \rrbracket$. We introduce two precision optimizations, taking advantage of implicit equalities represented by shared bi-cells. We first transform $*_{t} e_{1}$ and the dereferences in $e_{2}$ into sets of bi-cells $L$ and $R$, respectively. $R$ may be empty, as $e_{2}$ may be a constant expression. Then, we realize the cells in $L$ and $R$, using add-cell $b^{b}$. Let $\left\langle C_{0}, R_{0}\right\rangle$ be the updated environment. Two optimizations are possible, depending on $e_{1}, e_{2}, L$, and $R$.

Optimization 1: Assignment of shared bi-cells. If $e_{1}$ and $e_{2}$ are deterministic expressions, and if they evaluate to bi-cells that are all shared $\left(L \cup R \subseteq \widetilde{\mathrm{Cell}}^{2}\right)$, then Programs 1 and 2 write the same value to the same destination. We thus update shared destination bi-cells (in $L$ ), and remove any overlapping bi-cells. Formally:

$\mathbb{D}^{b} \llbracket *_{t} e_{1} \leftarrow e_{2} \rrbracket\langle C, R\rangle=\left\langle C_{0} \backslash \Omega\right.$, $\left.\left\{\rho_{\mid C_{0} \backslash \Omega}[\forall c \in L: c \mapsto v] \mid \rho \in R_{0}, v \in \mathbb{E}_{1}^{b} \llbracket e_{2} \rrbracket\left\langle C_{0}, R_{0}\right\rangle\right\}\right\rangle$,

where $\Omega \subseteq \mathcal{C}_{0} \backslash L$ is the set of (shared or projected) bi-cells overlapping elements of $L$. The choice of evaluating $\mathbb{E}_{1}^{b} \llbracket e_{2} \rrbracket$ (rather than $\left.\mathbb{E}_{2}^{b} \llbracket e_{2} \rrbracket\right)$ is arbitrary, as they are equal. Indeed, endianness $\alpha_{1}=\mathcal{L}$ is not used by $\mathbb{E}_{1}^{b} \llbracket e_{2} \rrbracket$, as all the necessary cells are materialized before evaluating expression $e_{2}$.

Optimization 2: Copy assignment. If the conditions for optimization 1 are satisfied, and if, in addition, $e_{2}=*_{t} e_{2}^{\prime}$, and both $*_{t} e_{1}$ and $*_{t} e_{2}^{\prime}$ evaluate to single bi-cells $(|L|=|R|=1)$, then we are dealing with a copy assignment. We may thus soundly copy a memory information from the source $\{l\}=L$ to the destination $\{r\}=R$, so as to further improve precision. We therefore create a copy of $r$, and any smaller bi-cell for the same bytes, to a corresponding bi-cell for the bytes of $l$. Newly created destination bi-cells have the sides and endiannesses of their sources. The environment is updated accordingly, to reflect equalities between sources and destinations.

\section{Value abstraction}

Connecting to numerical domains. We now rely on numeric abstractions to abstract further $\mathbb{D}^{b}$ into a computable abstract semantics $\mathbb{D}^{\sharp}$, resulting in an effective static analysis. Like [28, Sec. 5.2], our memory domain translates memory reads and writes into purely numerical operations on synthetic bi-cells, that are oblivious to the double semantics of double programs: each bi-cell is viewed as an independent numeric variable, and each numeric operation is carried out on a single bi-cell store, as if emanated from a single program. In particular, we notice that the transfer function for simple assignments $\mathbb{S}_{k}^{b} \llbracket *_{t} e_{1} \leftarrow e_{2} \rrbracket$ described in Sect. 3.5 has the form of that of an assignment in a purely numeric language, where bi-cells play the roles of the numeric variables. This property is 
a key motivation for the Cell domain and the extension presented in this paper. Bi-cells may thus be fed, as variables, to a numerical abstract domain for environment abstraction. Any standard numerical domain, such as polyhedra [12], may be used. Yet, as we aim at scaling to large programs, we restrict ourselves to combinations of efficient non-relational domains, intervals and congruences [18], together with a dedicated symbolic predicate domain.

We thus assume an abstract domain $\mathcal{D}_{C}^{\sharp}$ given, with concretization $\gamma_{C}$, for each bi-cell set $C \subseteq \mathcal{B}$ icell. It abstracts $\mathcal{P}(C \rightarrow \mathbb{Z}) \simeq \mathcal{P}\left(\mathbb{Z}^{|C|}\right)$, i.e., sets of points in a $|C|$-dimensional vector space. A cell of integer type naturally corresponds to a dimension in an abstract element. We also associate a distinct dimension to each cell with pointer type; it corresponds to the offset $o$ of a symbolic pointer $\langle V, o\rangle \in \mathcal{P}$ tr. In order to abstract fully pointer values, we enrich the abstract numeric environment with a map $P$ associating to each pointer cell the set of variables it may point to. Hence, the abstract domain becomes: $\mathcal{D}^{\sharp} \triangleq\left\{\left\langle C, R^{\sharp}, P\right\rangle \mid C \subseteq\right.$ Cell, $R^{\sharp} \in \mathcal{D}_{C}^{\sharp}, P \in P_{C} \rightarrow \mathcal{P}(\mathcal{V} \cup\{$ NULL, invalid $\left.\})\right\}$, where $P_{C} \subseteq C$ is the subset of bi-cells of pointer type. We refer to [28, Sec. 5.2] for a formal presentation of the concretization and the abstract operators.

Introducing a dedicated symbolic predicate domain. Recall Example 1 from Sect. 1. Various implementations are possible for the byte-swaps enforcing endian portability of software. Though Example 1 shows an implementation relying on type-punning, implementations relying on bitwise arithmetics are also commonplace. In addition, system-level software, such as [32], often rely on combinations of type-punning and bitwise arithmetics. Example 2 is a simplified instance of such programming idioms: as y has type unsigned char, yl0xff00 and $(y<<8) \mid 0 x f f$ represent the same 16-bit word in different endiannesses.

Example 2. Byte-wise equal memories in different endiannesses.

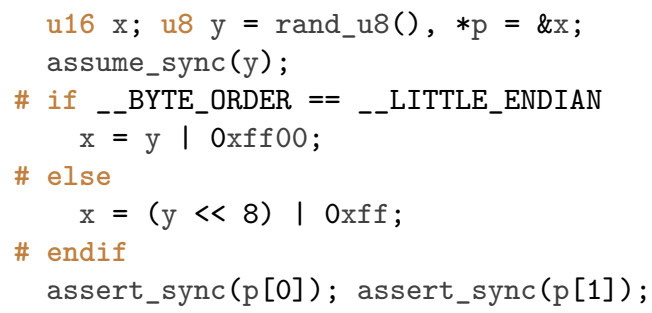

For a successful analysis of Example 2, the numerical domain must interpret bitwise arithmetic expressions precisely, and infer relations such as: the loworder (respectively high-order) byte of the little-endian (respectively big-endian) version of integer $\mathrm{x}$ is equal to $\mathrm{y}$. Then, the interpretation of dereferences of $\mathrm{p}$ by the memory domain introduces similar relations between cells, thanks to the bicell synthesize function. In this example, it infers that the little-endian version of the low-address (respectively high-address) byte cell in $\mathrm{x}$ is equal to the low-order (respectively high-order) byte of $\mathrm{x}$ - and the converse for big-endian. 
Predicate abstract domain. We use a domain based on pattern matching of expressions to detect arithmetic manipulations of byte values commonly implemented as bitwise arithmetics. It is not sufficient to match each expression independently, as computations are generally spread across sequences of statements. We need, in addition, to maintain some state that retains and propagates information between statements. We maintain this state in a predicate domain Pred $d^{\sharp} \triangleq C \rightarrow \mathcal{B}$ its, which maps each bi-cell $c \in C \subseteq \mathcal{B}$ icell to a syntactic expression $e$ in a language $\mathcal{B} i t s$, as a symbolic representation of predicate $c=e$.

$$
\begin{aligned}
& \text { Bits }::=\top \mid \text { Slice } \\
& \text { Slice }::=n|c| \overrightarrow{c[i, j}^{k} \mid(\text { Slice } \mid \text { Slice }) \quad(n \in \mathbb{Z}, c \in C, i, j, k \in \mathbb{N})
\end{aligned}
$$

$\top$ denotes the absence of information. Otherwise, a syntactic predicate expression may be either a bit-slice, or a bitwise OR of bit-slices. A bit-slice may be an integer constant $n$, a bi-cell $c$, or a slice expression $\overrightarrow{c[i, j}^{k}$ denoting the value obtained by shifting the bits of $c$ between $i$ and $j-1$ to position $k: \overrightarrow{c[i, j})^{k} \triangleq\lfloor(c$ $\left.\left.\bmod 2^{j}\right) / 2^{i}\right\rfloor \times 2^{k}$. Each term of a bitwise OR of bit-slices represents a interval of bits, e.g. $[k, k+j-i)$ for a term $\bar{c}[i, j)^{k}$. We assume that bit-intervals do not overlap: each bit from the result comes from a single cell or constant. The ordering is flat, based on syntactic predicate equality:

$$
X^{\sharp} \sqsubseteq^{\sharp} Y^{\sharp} \stackrel{\triangleq}{\Longleftrightarrow} \forall c \in C: X^{\sharp}(c)=Y^{\sharp}(c) \vee Y^{\sharp}(c)=\top
$$

An abstract element $X^{\sharp} \in \mathcal{P}$ red $d^{\sharp}$ denotes the set of environments that satisfy all the predicates in $X^{\sharp}$, where predicates are evaluated as expressions:

$$
\gamma_{\mathcal{P} \text { red }}\left(X^{\sharp}\right) \triangleq\left\{\rho \in C \rightarrow \mathbb{V} \mid \forall c \in C: X^{\sharp}(c)=\top \vee \rho(c) \in \mathbb{E} \llbracket X^{\sharp}(c) \rrbracket \rho\right\}
$$

We do not present the abstract operators in this paper. Like that of the related symbolic constant domain [26], they are based on symbolic propagation, and implement simple algebraic simplifications. They exhibit similar, near-linear time cost in our experiments.

Analysis of Example 2. Before line 8, three cells are synthesized by the memory domain: $C_{8}=\left\{x_{1}, x_{2}, y_{12}\right\}$, where $x_{1}=\langle x, 0, \mathbf{u 1 6}, \mathcal{L}, 1\rangle$ is the little-endian projected bi-cell of variable $\mathrm{x}, x_{2}=\langle x, 0, \mathbf{u 1 6}, \mathcal{B}, 2\rangle$ is the big-endian one, and $y_{12}=\langle\langle y, 0, \mathbf{u} \mathbf{8}, \mathcal{L}, 1\rangle,\langle y, 0, \mathbf{u} \mathbf{8}, \mathcal{B}, 2\rangle\rangle$ is a shared bi-cell.

- $y_{12}$ is created at line 2 , and represents the fact that variable $\mathrm{y}$ has the same value in the little- and big-endian versions.

- The transfer function for assignment of the symbolic predicate domain infers invariants $x_{1}=y_{12} \mid 65280$ from line 4 , and $x_{2}=255 \mid \vec{y} 12\left[0,8^{8}\right.$ from line 6 .

- Then, the dereferences of pointer $\mathrm{p}$ at line 8 are interpreted by the memory domain. Four more cells $\left\{x_{k}^{o} \mid(k, o) \in\{1,2\} \times\{0,1\}\right\}$ are added to the abstract environment, to denote the bytes of variable $\mathrm{x}$ in the little- and bigendian programs. More precisely, $x_{1}^{o}=\langle x, o, \mathbf{u} \mathbf{8}, \mathcal{L}, 1\rangle$, and $x_{2}^{o}=\langle x, o, \mathbf{u} \mathbf{8}, \mathcal{B}, 2\rangle$, at offsets $o \in\{0,1\}$. Following the bi-cell synthesize functions $\phi_{1}$ and $\phi_{2}$, these new bi-cells are added together with assumptions on their values. In practice, these assumptions are four tests, used by the memory domain to filter the abstract environment. These tests are $x_{1}^{o}=\operatorname{byte}\left(x_{1}, o\right)$, and $x_{2}^{o}=$ byte $\left(x_{1}, 1-o\right)$, for offsets $o \in\{0,1\}$, with byte $(n, k)=\left\lfloor n / 2^{8 k}\right\rfloor \bmod 2^{8}$. 
These tests are then interpreted by the numerical domain, and the symbolic predicate domain in particular, as $x_{1}^{o}=b y t e^{\prime}\left(x_{1}, o\right)$, and $x_{2}^{o}=b y t e^{\prime}\left(x_{2}, 1-o\right)$, with byte $(n, k)=\overrightarrow{n[8 k, 8 k+8)}^{0}$.

- Finally, the assertion line 8 is interpreted as tests $x_{1}^{0}=x_{2}^{0}$ and $x_{1}^{1}=x_{2}^{1}$ by the memory domain. The transfer function for tests in the symbolic predicate domain replaces all bi-cells with the symbolic expressions bound to them,

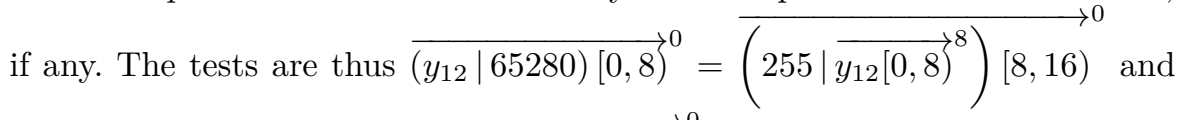

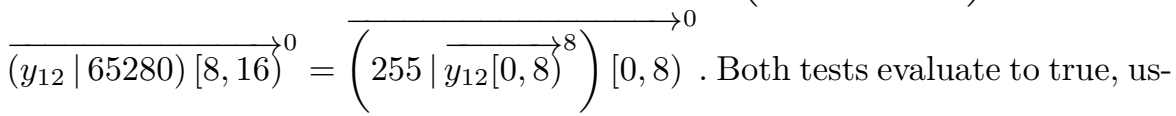
ing symbolic simplifications (and integer arithmetic computations) supported by the transfer function.

Hence, the assertions line 8 are proved correct: at the end of the program, the memories for variable $\mathrm{x}$ are byte-wise equal in the little and big-endian versions.

\section{Evaluation}

We implemented our analysis into the Mopsa platform [30,21] designed to support modular developments of precise static analyses for multiple languages and multiple properties. Our prototype is composed of 3,000 lines of OCaml: $45 \%$ for the memory abstraction, $36 \%$ for the symbolic predicate domain, and $19 \%$ for double program management and iterators. It leverages 31,000 lines (excluding parsers) of elementary functions of Mopsa: framework and utilities (64\%), generic iterators and numeric domains for analyses of all languages (11\%), specific iterators and memory domains for the $\mathrm{C}$ language (25\%). We have experimented our prototype on small idiomatic examples, open source software, and large industrial software. The analyses were run on a $3.4 \mathrm{GHz}$ Intel® Xeon® CPU.

\subsection{Idiomatic examples}

We first check the precision and robustness of our analysis against a collection of small double C programs (between 20 and 100 LOC), inspired by various implementations of byte-swaps in Linux drivers, POSIX htonl functions, and industrial software.

A set of 9 programs illustrate network data processing. These programs are similar to Example 1 of Sect. 1. They receive an integer from the network, increment it, and send over the result. Necessary byte-swaps are implemented for little-endian versions of these programs. Each example program implements a different byte-swapping technique on a 2, 4, or 8-byte integer: type-punning with pointer casts (like in Example 1), unions, or bitwise arithmetics. Refer to Examples 4, 5, and 6 in artifact [17] for the source codes. We also analyze Example 2 from Sect. 4 to demonstrate the efficiency of our symbolic predicate domain. 
Our prototype also handles floating-point data, which was omitted in the paper for the sake of conciseness. We developed small floating-point examples representative of industrial use-cases of Sect. 5.3. They include byte-swappings of simple or double precision floating-point numbers sent to or received from the network, on architectures where integers and floats are guaranteed to have the same byte-order. Type-punning is used to reinterpret floats as integers of the same size, which are byte-swapped using bitwise arithmetics. Also, a combination of type-punning and byte-swapping is used to extract exponents from double precision floats. The source codes of these Examples 8 and 9 is available in artifact [17]. All analyses run in less than $200 \mathrm{~ms}$ and report no false alarm.

\subsection{Open source benchmarks}

We then check the soundness, precision, and modularity of our analysis on three benchmarks based on open source software available on GitHub, with multiple commits for bug-fixes related to endianness portability. Refer to Examples 10, 11, and 12 in artifact [17] for relevant source codes excerpts. We analyze slices between 100 and 250 LOC, using primitives assume_sync and assert_sync for modular specifications of program parts.

Our first benchmark is an implementation of a tunneling driver [32] based on the Geneve [19] encapsulation network protocol, which uses big-endian integers as tunnel identifiers. The driver was introduced in the Linux kernel, and patched several times for endianness-related issues detected by SpARse [7]. Then, a performance optimization introduced a new endianness portability bug, which SPARSE failed to detect. It was fixed a year later. Our analysis soundly reports this bug, as well as previous issues detected by SPARSE. It reports no alarm on the fixed code. Our second benchmark is a core library of the mlx5 Linux driver [24] for ethernet and RDMA net devices [23]. We analyze a slice related to a patch, committed to fix an endianness bug introduced 3 years earlier, and undetected by SPARSE despite the use of relevant annotations. The fix turned out to be incomplete, and was updated 6 months later. Our analysis soundly reports

bugs on the two first versions, and no alarm on the third. Our third benchmark is extracted from a version of Squashfs [35], a compressed read-only filesystem for Linux, included in the LineageOS [34] alternative Android distribution. We analyze a slice related to a patch, committed to fix an endianness bug undetected by SPARSE due to a lack of type annotations. Our analysis soundly reports the bug, and no alarm on the fixed version. All the analyses run within 1 second.

\subsection{Industrial case study}

We analyzed two components of a prototype avionics application, developed at Airbus for a civil aircraft. This application is written in C, and primarily targets an embedded big-endian processor. Nonetheless, it must be portable to little-endian commodity hardware, as its source code is reused as part of a simulator used for functional verification of SCADE [4] models. The supplement to the applicable aeronautical standard [1] related to model-based development [2] 
mandates, in this case, that "an analysis should provide compelling evidence that the simulation approach provides equivalent defect detection and removal as testing of the Executable Object Code". Airbus, known to rely on formal methods for other verification objectives $[13,33,14,29,5]$, is currently considering the use of static analysis to verify this portability property.

Endianness is the main difference between the ABIs of the embedded computer and the simulator. We thus experimented our prototype analyzer on the modules of the application integrated to the simulator, to which we refer as A and S. Modules A and S are data-intensive reactive software, processing thousands of global variables, with very flat call graphs. Module A is in charge of acquiring and emitting data through aircraft buses. It is composed of about 1 million LOC, most of which generated automatically from a description of the avionics network. It handles integers, Booleans, single and double precision floats. The code features bounded loops, memcpys, pointer arithmetics, and type-punning with unions and pointer casts. It also uses bitwise arithmetics, among which several thousand byte-swaps related to endianness portability. Module $\mathrm{S}$ is in charge of the main applicative functions. It is composed of about 300,000 LOC, most of which generated automatically from SCADE models. It handles mostly Booleans and double precision floats. It features bounded loops and bitwise arithmetics, but no type-punning. The target application is required to meet its specifications for long missions. Analysis entry points contain loops with several million iterations to emulate this execution context.

Both analyses run in 5 abstract iterations. The analysis of A runs in 20.4 hours and uses 5.5 GB RAM. The analysis of S runs in 9.7 hours and uses 2.7 GB RAM. We worked with the development and simulation teams to analyze early prototypes, and incorporate findings into the development cycle. On current versions of both modules, both analyses report zero alarm related to endianness.

\section{Conclusion}

We presented a sound static analysis of endian portability for low-level C programs. Our method is based on abstract interpretation, and parametric in the choice of a numerical abstract domain. We first presented a novel concrete collecting semantics, relating the behaviors of two versions of a program, running on platforms with different endiannesses. Then we proposed a joint memory abstraction, able to infer equivalence relations between little- and big-endian memories. We introduced a novel symbolic predicate domain to infer relations between individual bytes of the variables in the two programs, which has nearlinear cost. We implemented a prototype static analyzer, able to scale to large real-world industrial software, with zero false alarms.

In future work, we aim at extending our analysis to further ABI-related properties, such as portability between different layouts of $\mathrm{C}$ types, or sizes of machine integers. We also anticipate that our bi-cell sharing approach will benefit the analysis of patches $[15,16]$ modifying $\mathrm{C}$ data-types, even if the two versions run under the same ABI. Finally, we are considering an industrial deployment 
of our endian portability analysis, as a means to address avionics certification objectives related to simulation fidelity, as mentioned in Sect. 5.3.

\section{References}

1. DO-178C: Software considerations in airborne systems and equipment certification (2011)

2. DO-331: Model-based development and verification supplement to DO-178C and DO-278A (2011)

3. AT \& T, The Santa Cruz Operation Inc.: System V application binary interface (1997)

4. Berry, G.: Scade: Synchronous design and validation of embedded control software. In: Ramesh, S., Sampath, P. (eds.) Next Generation Design and Verification Methodologies for Distributed Embedded Control Systems. pp. 19-33. Springer Netherlands, Dordrecht (2007)

5. Brahmi, A., Delmas, D., Essoussi, M.H., Randimbivololona, F., Atki, A., Marie, T.: Formalise to automate: deployment of a safe and cost-efficient process for avionics software. In: 9th European Congress on Embedded Real Time Software and Systems (ERTS 2018). Toulouse, France (Jan 2018), https://hal. archives-ouvertes.fr/hal-01708332

6. Brevnov, E., Domeika, M., Loenko, M., Ozhdikhin, P., Tang, X., Willkinson, H.: Bec: Bi-endian compiler technology for porting byte order sensitive applications $16(2012)$

7. Brown, N.: Sparse: a look under the hood (2016), https://1wn.net/Articles/ 689907/

8. Chevalier, M.: Proving the Security of Software-Intensive Embedded Systems by Abstract Interpretation. Ph.D. thesis, Université PSL (Nov 2020)

9. Chevalier, M., Feret, J.: Sharing ghost variables in a collection of abstract domains. In: Beyer, D., Zufferey, D. (eds.) Verification, Model Checking, and Abstract Interpretation. pp. 158-179. Lecture Notes in Computer Science, Springer International Publishing (2020)

10. Cohen, D.: On holy wars and a plea for peace. Computer 14(10), 48-54 (1981). https://doi.org/10.1109/C-M.1981.220208

11. Cousot, P., Cousot, R.: Abstract interpretation: A unified lattice model for static analysis of programs by construction or approximation of fixpoints. In: POPL'77. pp. 238-252. ACM (Jan 1977)

12. Cousot, P., Halbwachs, N.: Automatic discovery of linear restraints among variables of a program. In: POPL'78. pp. 84-97. ACM (1978)

13. Delmas, D., Souyris, J.: Astrée: from research to industry. In: SAS'07, LNCS, vol. 4634, pp. 437-451. Springer (Aug 2007)

14. Delmas, D., Goubault, E., Putot, S., Souyris, J., Tekkal, K., Védrine, F.: Towards an industrial use of fluctuat on safety-critical avionics software. In: Alpuente, M., Cook, B., Joubert, C. (eds.) FMICS. Lecture Notes in Computer Science, vol. 5825, pp. 53-69. Springer (2009)

15. Delmas, D., Miné, A.: Analysis of Program Differences with Numerical Abstract Interpretation. In: PERR 2019. Prague, Czech Republic (Apr 2019)

16. Delmas, D., Miné, A.: Analysis of Software Patches Using Numerical Abstract Interpretation. In: Chang, B.Y.E. (ed.) Proc. of the 26th International Static Analysis Symposium (SAS'19). Lecture Notes in Computer Science, vol. 11822, pp. 225-246. Bor-Yuh Evan Chang, Springer, Porto, Portugal (Oct 2019) 
17. Delmas, D., Ouadjaout, A., Miné, A.: Artifact for Static Analysis of Endian Portability by Abstract Interpretation (2021). https://doi.org/10.5281/zenodo.5206794

18. Granger, P.: Static analysis of arithmetic congruences. Int. Journal of Computer Mathematics 30, 165-199 (1989)

19. Gross, J., Ganga, I., Sridhar, T.: Geneve: Generic network virtualization encapsulation. RFC 8926, RFC Editor (November 2020)

20. ISO/IEC JTC1/SC22/WG14 working group: C standard. Tech. Rep. 1124, ISO \& IEC (2007)

21. Journault, M., Miné, A., Monat, R., Ouadjaout, A.: Combinations of reusable abstract domains for a multilingual static analyzer. In: Proc. of the 11th Working Conference on Verified Software: Theories, Tools, and Experiments (VSTTE19). Lecture Notes in Computer Science (LNCS), vol. 12031, pp. 1-18. Springer (Jul 2019)

22. Kápl, R., Parízek, P.: Endicheck: Dynamic analysis for detecting endianness bugs. In: Biere, A., Parker, D. (eds.) Tools and Algorithms for the Construction and Analysis of Systems. pp. 254-270. Springer International Publishing, Cham (2020)

23. Mahameed, S.: Mellanox, mlx5 rdma net device support (2017), https://1 wn.net/ Articles/720074/

24. Mellanox Technologies: mlx5 core library (2020), https://github.com/torvalds/ linux/tree/master/drivers/net/ethernet/mellanox/mlx5/core

25. Miné, A.: Field-sensitive value analysis of embedded $\mathrm{C}$ programs with union types and pointer arithmetics. In: Proc. of the ACM SIGPLAN/SIGBED Conf. on Languages, Compilers, and Tools for Embedded Systems (LCTES'06). pp. 54-63. ACM (June 2006)

26. Miné, A.: Symbolic methods to enhance the precision of numerical abstract domains. In: Proc. of the 7th Int. Conf. on Verification, Model Checking, and Abstract Interpretation (VMCAI'06). LNCS, vol. 3855, pp. 348-363. Springer (Jan 2006)

27. Miné, A.: Abstract domains for bit-level machine integer and floating-point operations. In: Proc. of the 4th Int. Workshop on Invariant Generation (WING'12). p. 16. No. HW-MACS-TR-0097, Computer Science, School of Mathematical and Computer Science, Heriot-Watt University, UK (Jun 2012)

28. Miné, A.: Static analysis by abstract interpretation of concurrent programs. Tech. rep., École normale supérieure (May 2013)

29. Miné, A., Delmas, D.: Towards an industrial use of sound static analysis for the verification of concurrent embedded avionics software. In: Proc. of the 15th International Conference on Embedded Software (EMSOFT'15). pp. 65-74. IEEE CS Press (Oct 2015)

30. Miné, A., Ouadjaout, A., Journault, M.: Design of a Modular Platform for Static Analysis. In: The Ninth Workshop on Tools for Automatic Program Analysis (TAPAS'18). Fribourg-en-Brisgau, Germany (Aug 2018), https://hal. sorbonne-universite.fr/hal-01870001

31. Nita, M., Grossman, D.: Automatic transformation of bit-level C code to support multiple equivalent data layouts. In: Hendren, L.J. (ed.) Compiler Construction, 17th International Conference, CC 2008, Held as Part of the Joint European Conferences on Theory and Practice of Software, ETAPS 2008, Budapest, Hungary, March 29 - April 6, 2008. Proceedings. Lecture Notes in Computer Science, vol. 4959, pp. 85-99. Springer (2008)

32. Red Hat, Inc.: Generic network virtualization encapsulation (2017), https:// github.com/torvalds/linux/blob/master/drivers/net/geneve.c 
33. Souyris, J., Wiels, V., Delmas, D., Delseny, H.: Formal verification of avionics software products. pp. 532-546 (2009)

34. The LineageOS Project: Lineageos (2020), https://github.com/Lineage0S/

35. The Squashfs Project: Squashfs (2020), https://github.com/Lineage0S/ android_kernel_sony_msm8960t/tree/lineage-18.1/fs/squashfs 